\title{
Effet de l'incorporation de farines de soja et de poisson dans l'aliment sur la croissance et les enzymes digestives de Penaeus vannamei
}

\author{
François Galgani $^{(1),(3)}$, Hubert J. Ceccaldi ${ }^{(2)}$ et AQUACOP(1) \\ (1) Équipe du Centre Océanologique du Pacifique, IFREMER, BP n ${ }^{\circ} 7004$, Taravao, Tahiti (Polynésie française). \\ (2) Laboratoire EPHE de Biochimie des Invertébrés Marins, Centre d'Océanologie de Marseille, \\ URA-CNRS $n^{\circ}$ 41, 13007 Marseille (France). \\ (3) Adresse actuelle : IFREMER, Centre de Nantes, BP $n^{\circ}$ 1049, 44037 Nantes Cedex 01 (France).
}

Reçu le 12 avril 1988, accepté le 8 juin 1988.

The effect of the incorporation of soya bean and fish meals in the diet on the growth and digestive enzymes of Penaeus vannamei.

Galgani F., H. J. Ceccaldi, AQUACOP. Aquat. Living Resour., 1988, 1, 181-187.

Abstract

In order to improve the formulation of diets fed to reared Penaeus vannamei, the effects of soya bean and fish meals on the growth, survival, and the characteristics of the hepatopancreas and digestive enzymes of the species were studied. Four soya bean meal concentrations $(0,5,15,40 \%)$, four fish meal concentrations $(0,5,15,30 \%)$, two forms of soya bean meal and three fish meals of different geographical origin were tested. Experimental conditions were as follows: ten individuals were placed in each tank $\left(0.5 \mathrm{~m}^{2}\right)$ and two tanks were subjected to each treatment. Food was given twice a day $(08-09.00 \mathrm{~h}$ and $19.00 \mathrm{~h})$ at $10 \%$ of the initial weight each day. For biochemical analyses, nine individuals per treatment were used for the determination of the hepatosomatic ratio, proteins, proteases, amylases and trypsin. The results show that a high level of soya bean or fish meals in the diet strongly depresses growth without any apparent effect on the digestive enzymes or the hepatopancreas parameters. No effect of soya bean meal type or geographical origin of the fish meal was detected. The results suggest that the incorporation of both soya bean and fish meals in the diet should be restricted to low concentrations and only with the aim of diversifying the protein sources.

Keywords : Aquaculture, nutrition, Penaeidae, soya bean, fish meal, digestive enzymes.

Résumé

Afin d'améliorer la formulation des aliments composés destinés à la croissance de Penaeus vannamei, nous avons étudié l'effet de l'incorporation de farine de soja et de farine de poisson sur la croissance, la survie, les caractéristiques de l'hépatopancréas et les enzymes de cette espèce. Quatre concentrations de farine de soja $(5,10,20,40 \%)$, quatre concentrations de farine de poisson $(0,5,15,30 \%)$ deux formes de farine de soja et trois farines de poissons différentes suivant leur origine géographique ont été successivement testées. La nourriture (10\% du poids initial) a été distribuée deux fois par jour (8$9 \mathrm{~h}$ et $19 \mathrm{~h}$ ). Les rapports hépatosomatiques, les protéines, les protéases, les amylases et la trypsine ont été déterminés sur neuf individus par traitement. Les résultats montrent qu'une forte incorporation de farine de soja ou de farine de poisson a pour effet de diminuer la croissance des animaux. Cette diminution est liée à une baisse de l'ingestibilité des aliments. Les enzymes digestives ne sont pas affectées. Les résultats montrent par ailleurs que l'origine géographique d'une source de poisson et le traitement subi par la farine de soja et la farine de poisson d'autre part, n'affectent pas les performances zootechniques et les enzymes digestives. L'ensemble des résultats permet de conclure que l'incorporation de soja ou de farine de poisson dans l'aliment doit être réalisée à faible taux, et ce, uniquement dans le but de diversifier les sources de protéines.

Mots-clés : Aquaculture, nutrition, Pénéidés, soja, farine de poisson, enzymes, enzymes digestives. 


\section{INTRODUCTION}

Le tourteau de soja et la farine de poisson sont les ingrédients les plus utilisés en alimentation animale en général et dans l'alimentation des crustacés d'élevage en particulier (Ceccaldi, 1986). Les résultats obtenus précédemment ont montré que le taux de croissance pouvait varier suivant l'origine de la farine considérée mais aussi suivant le traitement subi avant l'incorporation dans l'aliment. Par ailleurs, l'emploi de certaines farines conduit quelquefois à de mauvaises croissances alors que théoriquement la teneur en protéines et l'équilibre aminoacide sont assurés. Divers auteurs ont montré que parmi les ingrédients de base utilisés dans les aliments destinés à l'aquaculture, certains se caractérisent par la présence de facteurs antinutritionnels ayant un effet néfaste sur la croissance des organismes. C'est le cas d'un facteur inhibiteur de la trypsine qui existe dans le soja (Rackis, 1974; Wilson et Poe, 1985). D'une manière générale, la présence d'inhibiteur trypsique dans l'aliment se traduit par une hypertrophie du pancréas, une baisse des activités enzymatiques et une réduction de la croissance (Rackis, 1974). La nature des enzymes digestives des crustacés commence à être mieux connue (Gibson et Barker, 1979; Dall et Moriarty, 1983; Galgani, 1987) ainsi que leurs variations au cours des cycles physiologiques (Van Wormhoudt, 1980). La plupart des travaux concernent les aspects comparatifs de la digestion. Un certain nombre d'études intéressent cependant directement l'aquaculture. Il s'agit de l'étude du mécanisme d'adaptation de l'équipement enzymatique au régime alimentaire (Hoyle, 1973; Van Wormhoudt et al., 1981; Maugle et al., 1982) ainsi que la caractérisation et l'évaluation de la qualité d'un ingrédient particulier (Lee et al., 1984; Galgani et al., sous presse). L'objet du présent travail est d'une part de préciser le taux optimal d'incorporation du tourteau de soja et de la farine de poisson. D'autre part, nous avons comparé trois farines de poisson différentes suivant l'origine géographique et deux sources de soja différentes suivant l'origine géographique et deux sources de soja différentes selon le traitement subi avant l'incorporation dans l'aliment. Enfin, l'activité des enzymes digestives a été mesurée afin de préciser les variations de l'équipement enzymatique liées au régime alimentaire.

\section{MATÉRIELS ET MÉTHODES}

Quatre expériences différentes ont été réalisées. Dans les deux premières expériences, l'effet respectif du taux d'incorporation du tourteau de soja et de la farine de poisson sur la croissance est mesuré. Dans une troisième expérience, l'effet de deux sources différentes de soja sur la croissance est comparé. Enfin dans une quatrième expérience, trois sources de farines de poisson d'origine géographique différente sont comparées.

\section{Animaux et dispositif expérimental}

Les crevettes Penaeus vannamei sont issues de pontes réalisées à partir de géniteurs captifs dans les conditions décrites par AQUACOP (1977).

Les animaux sont répartis dans des bacs de 5001 ( $1 \mathrm{~m}$ de long $\times 1 \mathrm{~m}$ de large $\times 0,5 \mathrm{~m}$ de profondeur) à une densité initiale de $10 / \mathrm{m}^{2}$. L'eau est renouvelée 10 fois par jour, la photopériode est de 12/12 et l'éclairage est réalisé à l'aide de lumière naturelle et de lampes à incandescence. Chaque régime est testé sur deux bacs expérimentaux.

\section{Aliments \\ Les aliments sont préparés selon la méthode décrite par AQUACOP (1979). (La composition proximale}

Tableau 1. - Composition proximale des farines expérimentées $(\%$ du poids sec).

Proximal composition of tested meals ( $\%$ of the dry weight).

\begin{tabular}{lccc}
\hline & Protéines & Lipides & Cendres \\
\hline Farine de soja & 50 & 1,5 & 6,6 \\
Concentré de soja & 64 & 0,5 & 5,6 \\
Farine de poisson & & & \\
- NSM & 72 & 10 & 10 \\
- Lorientaise & 60 & 9,5 & 22 \\
- Danoise & 66 & 8 & 15 \\
\hline
\end{tabular}

des ingrédients est donnée dans le tableau 1). La composition des aliments est donnée au chapitre " résultats 》. Le mélange protéique est constitué de $50 \%$ de concentré de protéines solubles de poissons, $31 \%$ de farine de crevette, $14 \%$ de levure lactique et de $5 \%$ de farine de sang. Le tourteau de soja et le concentré de soja proviennent respectivement des compagnies Barlas (USA) et Staley MFG (USA). Les farines de poisson norvégienne (Norseamind) et lorientaise proviennent de la société Sopropêche. La farine de poisson danoise nous a été fournie par l'INRA (Jouy-en-Josas, France). La composition spécifique des farines de poissons n'est pas connue.

\section{Contrôle des élevages}

Les expériences sont réalisées en double sur une période de 28 jours. La distribution biquotidienne des aliments est réalisée entre 8 et 9 heures le matin et 19 heures le soir. La ration journalière est de $10 \% \mathrm{du}$ poids initial. Tous les matins, la consommation est estimée, les exuvies et les animaux morts sont prélevés.

\section{Méthodes d'analyses}

En fin d'expérience, trois hépatopancréas par bac expérimental (6 par traitement) sont prélevés entre 12 et 13 heures, pesés et lyophilisés. La préparation d'extraits enzymatiques à partir des hépatopancréas est réalisée suivant une méthode décrite précédemment (Galgani et al., 1987). Chaque hépatopancréas 
Tableau 2. - Influence de l'incorporation de soja dans l'aliment sur les performances et les enzymes digestives de Penaeus vannamei. Effect of soya bean meal concentration on the growth and digestive enzymes of Penaeus vannamei.

(A) Composition des aliments

\begin{tabular}{lcccc}
\hline \multicolumn{1}{c}{ Aliment } & 1 & 2 & 3 & 4 \\
\hline \% Soja & 5 & 10 & 20 & 40 \\
\hline Mélange protéique & 41 & 37,4 & 30,1 & 15,6 \\
Soja (tourteau) & 5 & 10 & 20 & 40 \\
Farine de froment & 21,5 & 20,07 & 17,3 & 11,9 \\
Cellulose & 3,25 & 3,25 & 3,25 & 3,25 \\
Gluten de blé & 15 & 15 & 15 & 15 \\
CaCO & 0,5 & 0,5 & 0,5 & 0,5 \\
NaHPO & 3,5 & 3,5 & 3,5 & 3,5 \\
Lécithine de soja & 1,75 & 1,75 & 1,75 & 1,75 \\
Huile rouge $(*)$ & 1,75 & 1,75 & 1,75 & 1,75 \\
Prémix vitaminique & 3,5 & 3,5 & 3,5 & 3,5 \\
Liagel (**) & 3 & 3 & 3 & 3 \\
\hline Protéines $(\%)$ & 44 & 43,3 & 43,1 & 43 \\
Lipides $(\%)$ & 6,1 & 6,4 & 6,1 & 6 \\
Eau & 9,2 & 4,1 & 9 & 13 \\
Cendres $(\%)$ & 18 & 13 & 13 & 22 \\
\hline
\end{tabular}

(*) Huile de foie de Gadidés. (**) Amidon prégélatinisé.

\begin{tabular}{|c|c|c|c|c|c|}
\hline \multicolumn{6}{|c|}{ (B) Résultats } \\
\hline Aliment & 1 & & 2 & 3 & 4 \\
\hline Poids initial & $2,35(0,17)$ & $2,23(0,19)$ & $2,28(0,19)$ & $2,35(0,18)$ & \\
\hline Poids final & $5,28(0,3)$ & $5,12(0,3)$ & $4,99(0,3)$ & $4,42(0,3)$ & \\
\hline Accroissement & 2,93 & 2,84 & 2,68 & 2,07 & \\
\hline Mortalité & $13 \%$ & $8 \%$ & $8 \%$ & $8 \%$ & \\
\hline Nombre de mues $/ 28 \mathrm{j}$ & 51 & 48 & 46 & 41 & \\
\hline Taux de conversion & 2 & 2 & 2 & 2 & \\
\hline RHS $\left(^{*}\right)$ frais & $4,32(0,32)$ & $4,28(0,41)$ & $4,36(0,36)$ & $4,5(0,47)$ & \\
\hline RHS sec & $1,53(0,22)$ & $1,75(0,31)$ & $1,5 \quad(0,2)$ & $1,77(0,38)$ & \\
\hline Protéines (mg/g HP) & $17,5 \quad(1,2)$ & $14,8 \quad(2)$ & $16 \quad(1,59)$ & $18 \quad(2,5)$ & \\
\hline Amylases $\left({ }^{* *}\right)(\mathrm{A})$ & $9,8 \quad(1,3)$ & $7,6 \quad(0,5)$ & $9,3 \quad(0,8)$ & $9,5(2,1)$ & \\
\hline Protéases $(* *)(\mathrm{P})$ & $8,9 \quad(1,0)$ & $7,9 \quad(0,8)$ & $8,4 \quad(1,3)$ & $7,9 \quad(0,9)$ & \\
\hline Trypsine $\left({ }^{* *}\right)(\mathrm{T})$ & $9,7 \quad(1,6)$ & $8,7 \quad(1,3)$ & $7,9 \quad(1,6)$ & $8,9 \quad(1,8)$ & \\
\hline $\mathrm{A} / \mathrm{P}$ & $1,1 \quad(0,15)$ & $1,0 \quad(0,14)$ & $1,12(0,19)$ & $1,18(0,15)$ & \\
\hline $\mathrm{T} / \mathbf{P}$ & $1,1 \quad(0,1)$ & $1,1 \quad(0,26$ & $1,0 \quad(0,18)$ & $1,14(0,24)$ & \\
\hline $\begin{array}{l}\text { (*) RHS: Rapport hép } \\
(* *) \text { Activités spécifiqu } \\
(\text { ) Intervalle de confi }\end{array}$ & rotéines d' & topancréas. & & & \\
\hline
\end{tabular}

est extrait par $5 \mathrm{~cm}^{3}$ de tampon phosphate $10 \mathrm{mmol} \mathrm{pH} 7$. Les protéines solubles sont dosées par la méthode de Lowry et al. (1951). Les activités protéasiques, amylasiques et trypsiques sont mesurées au spectrophotomètre suivant respectivement les procédures de Charney et Tomarelli (1947), Bernfeld (1955) et Hummel (1959). Les mesures sont effectuées à $20^{\circ} \mathrm{C}$ en utilisant l'azocoll (protéases), l'amidon (amylase) et le tosyl-arginine methyl ester (TAME) comme substrats.

\section{Méthodes statistiques}

Les comparaisons statistiques ont été réalisées en utilisant le test de Student.

\section{RÉSULTATS}

\section{Expérience 1}

Dans le cadre de cette expérience, l'effet d'une forte incorporation de tourteau de soja dans l'aliment sur la croissance de Penaeus vannamei est testé. Quatre aliments contenant respectivement $5,10,20$ et $40 \%$ de tourteau de soja sont comparés. La composition exacte des aliments et les résultats sont présentés dans le tableau 2.

Les résultats montrent que l'incorporation de fortes quantités de farine de soja provoque une diminution de l'accroissement pondéral des animaux. En effet, l'accroissement mensuel varie de $2,93 \mathrm{~g} \mathrm{(5 \%} \mathrm{de} \mathrm{soja}$ dans l'aliment) à $2,07 \mathrm{~g}$ ( $40 \%$ de soja dans l'aliment $)$. 
Tableau 3. - Influence de l'incorporation de farine de poisson dans l'aliment sur les performances et les enzymes digestives de Penaeus vannamei.

Effect of fish meal concentration on the growth and digestive enzymes of Penaeus vannamei.

(A) Composition des aliments

\begin{tabular}{lcccc}
\hline \multicolumn{1}{c}{ Aliment } & 1 & 2 & 3 & 4 \\
\hline Farine de poisson & 0 & 5 & 15 & 30 \\
Mélange protéique & 41 & 35,5 & 24,8 & 8,7 \\
Soja & 5 & 5 & 5 & 5 \\
Farine de poisson & 21,5 & 22 & 23 & 24 \\
Cellulose & 3,25 & 3,25 & 3,25 & 3,25 \\
Gluten de blé $_{\text {CaCO }_{3}}$ & 15 & 15 & 15 & 15 \\
NaHPO $_{4}$ & 0,5 & 0,5 & 0,5 & 0,5 \\
Lécithine de soja & 3,5 & 3,5 & 3,5 & 3,5 \\
Huile rouge & 1,75 & 1,75 & 1,75 & 1,75 \\
Prémix vitaminique & 1,75 & 1,75 & 1,75 & 1,75 \\
Liagel & 3,5 & 3,5 & 3,5 & 3,5 \\
\hline Protéines (\%) & 3 & 3 & 3 & 3 \\
Lipides $(\%)$ & 44 & 43,2 & 43,2 & 43,3 \\
Eau (\%) & 6,1 & 5,5 & 6,4 & 7,7 \\
Cendres $(\%)$ & 9,2 & 14 & 10 & 12,7 \\
\hline
\end{tabular}

(B) Résultats

\begin{tabular}{|c|c|c|c|c|}
\hline Aliment & 1 & & 2 & 4 \\
\hline Poids initial & $2,35(0,17)$ & $2,26(0,17)$ & $2,26(0,17)$ & $2,26(0,3)$ \\
\hline Poids final & $5,28(0,3)$ & $5,0 \quad(0,25)$ & $4,93(0,2)$ & $4,34(0,25)$ \\
\hline Accroissement & 2,93 & 2,75 & 2,66 & 2,08 \\
\hline Mortalité & $13 \%$ & $0 \%$ & $0 \%$ & $5 \%$ \\
\hline Nombre de mues $/ 28 \mathrm{j}$ & 51 & 66 & 55 & 42 \\
\hline Taux de conversion & 2 & 2,05 & 1,95 & 1,9 \\
\hline RHS $\left({ }^{*}\right)$ frais & $4,3 \quad(0,4)$ & $4,9 \quad(0,5)$ & $5,1 \quad(0,5)$ & $5,4 \quad(0,6)$ \\
\hline RHS sec & $1,5 \quad(0,2)$ & $1,8 \quad(0,3)$ & $1,9 \quad(0,3)$ & $2,2 \quad(0,2)$ \\
\hline Protéines $(\mathrm{mg} / \mathrm{g})$ & $17,5(1,2)$ & $14,5 \quad(1,1)$ & $15,5 \quad(1,8)$ & $15,0 \quad(2,0)$ \\
\hline Amylases $(* *)(\mathrm{A})$ & $9,8 \quad(1,3)$ & $11,2 \quad(1,1)$ & $10,6 \quad(1,6)$ & $12,2 \quad(1,9)$ \\
\hline Protéases $(* *)(\mathrm{P})$ & $8,9 \quad(1,0)$ & $8,5 \quad(0,5)$ & $8,6 \quad(0,9)$ & $8,7 \quad(0,7)$ \\
\hline Trypsine $\left({ }^{* *}\right)(\mathrm{T})$ & $9,6 \quad(1,6)$ & $11,1 \quad(1,57)$ & $11,4 \quad(0,25)$ & $8,4 \quad(2,5)$ \\
\hline $\mathrm{A} / \mathrm{P}$ & $1,11(0,15)$ & $1,32(0,18)$ & $1,31(0,3)$ & $1,4 \quad(0,25)$ \\
\hline$T / P$ & $1,08(0,07)$ & $1,31(0,24)$ & $1,23(0,17)$ & $0,97(0,27)$ \\
\hline
\end{tabular}

(*) RHS : Rapport hépatosomatique.

${ }^{(* *)}$ Activités spécifiques: unités/mg de protéines d'hépatopancréas.

( ) Intervalle de confiance $(p=0,05)$.

Quelle que soit la concentration de soja dans l'aliment, peu de différences sont observées pour la mortalité et le rythme de mue. Les valeurs de taux de conversion sont identiques et égales à 2 . Par ailleurs, le tableau 2(B) montre qu'il n'y a pas d'effet du taux d'incorporation de soja sur les valeurs des rapports hépatosomatiques (RHS). Afin de détecter les modifications de l'équipement enzymatique au niveau moléculaire sur la concentration en protéines de la glande digestive, les valeurs d'activités enzymatiques spécifiques de type protéase, amylase et trypsine ont été mesurées. Les résultats montrent que les taux d'activités sont constants quel que soit le régime alimentaire. Il en est de même pour les valeurs des ratios trypsine/ protéase $(\mathrm{T} / \mathrm{P})$ et amylase/protéase $(\mathrm{A} / \mathrm{P})$.

\section{Expérience 2}

Dans cette expérience, quatre régimes contenant respectivement $0,5,15$ et $30 \%$ de farine de poisson sont donnés dans le tableau 3. Comme pour la farine de soja, l'incorporation de fortes quantités de farine de poisson provoque une diminution des performances. En effet, de 0 à $30 \%$ de farine de poisson pour l'aliment, l'accroissement mensuel diminue de $2,93 \mathrm{~g} /$ mois à $2,08 \mathrm{~g}$. Les différences de mortalité ne sont pas liées au taux d'incorporation de la farine de poisson. Pour les quatres régimes alimentaires, le taux de conversion ne varie pas. Par contre la valeur du RHS mesurée sur les hépatopancréas frais ou secs augmente de manière significative lorsque la quantité de farine augmente. 
$\mathrm{Au}$ niveau moléculaire, aucune modification du spectre des protéases n'est observée avec une valeur constante de l'activité spécifique des protéases, de la trypsine et du rapport trypsine/protéase $(\mathrm{T} / \mathrm{P})$. Pour ce qui concerne l'amylase, on observe une augmentation significative de l'activité spécifique de l'enzyme ainsi qu'une variation du rapport amylase/protéase $(\mathrm{A} / \mathrm{P})$.

\section{Expérience 3}

Au cours de cette expérience, nous avons comparé l'influence du tourteau de soja et du concentré de soja sur la croissance de Penaeus vannamei. La composition des aliments testés et les résultats sont présentés

Tableau 4. - Influence comparée du tourteau de soja et du concentré de soja sur la croissance de Penaeus vannamet.

Comparison of two diets containing respectively soya bean meal and soya bean concentrate.

(A) Composition des aliments

\begin{tabular}{lcc}
\hline \multicolumn{1}{c}{ Aliments } & $\begin{array}{c}\text { Tourteau } \\
\text { de soja }\end{array}$ & $\begin{array}{c}\text { Concentré } \\
\text { de soja }\end{array}$ \\
\hline Tourteau de soja & 20 & - \\
Concentré de soja & - & 20 \\
Mélange protéique & 30 & 30 \\
Farine de froment & 17,5 & 17,5 \\
Cellulose & 3,5 & 3,5 \\
Gluten de blé & 15 & 15 \\
CaCO & 0,5 & 0,5 \\
NaHPO $_{4}$ & 3,5 & 3,5 \\
Lécithine de soja & 1,75 & 1,75 \\
Huile rouge & 3,5 & 3,5 \\
Prémix vitaminique & 3,5 & 3,5 \\
Liagel & 3 & 3 \\
\hline
\end{tabular}

(B) Résultats

\begin{tabular}{lcc} 
Poids initial & $5,32(0,47)$ & $5,4(0,47)$ \\
Poids final & $8,65(0,55)$ & $9,01(0,65)$ \\
Accroissement & 3,34 & 3,61 \\
Mortalité & 0 & 0 \\
Nombre de mues $/ 28 \mathrm{j}$ & 55 & 58 \\
Taux de conversion & 3,4 & 2,9 \\
\hline ( Intervalle de confiance $(p=0,05)$. & &
\end{tabular}

dans le tableau 4. Seules de légères différences non statistiquement significatives de l'accroissement mensuel du taux de conversion sont observées entre les animaux nourris de tourteau de soja d'une part et de concentré de soja d'autre part. Les mortalités et le nombre de mues observés pour 28 jours d'expérience sont très proches pour les deux aliments testés.

\section{Expérience 4}

Dans cette dernière expérience, trois farines de poisson qui différent suivant leur origine et donc suivant leur composition intrinsèque sont incorporées dans trois aliments et testées. La composition exacte des aliments et les résultats des tests zootechniques sont présentés dans le tableau 5. Pour ce qui concerne l'accroissement mensuel des animaux, une légère différence non statistiquement significative est observée suivant le régime alimentaire utilisé. Pour les trois régimes considérés, aucune mortalité n'est observée durant l'expérience. Par ailleurs, un très fort nombre de mue a été observé chez les animaux nourris à l'aide de farine danoise.

Tableau 5. - Influence comparée de trois farines de poissons sur les performances de croissance de Penaeus vannamei.

Effect of three fish meals on the growth of Penaeus vannamei.

(A) Composition des aliments

\begin{tabular}{lccc}
\hline \multicolumn{1}{c}{ Aliment } & $\begin{array}{c}\text { Farine } \\
\text { de Norvège }\end{array}$ & $\begin{array}{c}\text { Farine } \\
\text { lorientaise }\end{array}$ & $\begin{array}{c}\text { Farine } \\
\text { danoise }\end{array}$ \\
\hline Farine norvégienne & 15 & & \\
Farine lorientaise & & 15 & \\
Farine danoise & 25 & 25 & 15 \\
Mélange protéique & 5 & 5 & 25 \\
Soja & 22 & 22 & 5 \\
Farine de froment & 3 & 3 & 22 \\
Cellulose & 16 & 16 & 3 \\
Gluten de blé & 0,5 & 0,5 & 16 \\
CaCO & 3,5 & 3,5 & 0,5 \\
NaHPO & & 1,75 & 3,5 \\
Lécithine de soja & 1,75 & 1,75 & 1,75 \\
Huile rouge & 1,75 & 3,5 & 1,75 \\
Prémix vitaminique & 3,5 & 3 & 3,5 \\
Liagel & 3 & & 3 \\
\hline
\end{tabular}

(B) Résultats

$\begin{array}{lccc}\text { Poids initial } & 1,55(0,14) & 1,48(0,13) & 1,49(0,15) \\ \text { Poids final } & 3,71(0,5) & 3,74(0,45) & 3,82(0,50) \\ \text { Accroissement } & 2,16 & 2,27 & 2,33 \\ \text { Nombre de mues/28 j } & 41 & 47 & 58 \\ \text { Taux de conversion } & 2,3 & 2,1 & 1,8 \\ \text { Mortalité } & 0 & 0 & 0\end{array}$

( ) Intervalle de confiance $(p=0,05)$.

\section{DISCUSSION}

Dans cette étude, nous avons étudié l'effet de l'incorporation de farine de soja et de farine de poisson sur les performances de croissance et les enzymes digestives de Penaeus vannamei. Des travaux précédents (Venkatahramiah, 1975) ont montré que la présence de matière végétale était essentielle pour augmenter l'efficacité des aliments et la survie des animaux. L'utilisation de farine de soja dans l'alimentation des crustacés est très controversée. Kanazawa (1970) évalue la protéine de soja comme une très bonne source protéique, Fennucci (1982) et Galgani et al. (1987) ont cependant montré qu'il existait de meilleures sources protéiques. C'est la cas en particulier de protéines de crustacés ou de calmar et d'une manière plus générale de protéines d'origine marine. Les résultats obtenus chez Penaeus vannamei montrent qu'une trop forte incorporation de soja a pour effet de baisser la croissance. On peut déterminer une valeur d'incorporation au-delà de laquelle la croissance est 
affectée. L'incorporation de farine de soja paraît intéressante toutefois dans la mesure où elle permet de diversifier les sources protéiques, la diversification étant nécessaire pour obtenir une bonne croissance (Shigueno, 1972; Boghen et Castell, 1981; Galgani, 1987).

Des travaux réalisés sur divers animaux (Rackis, 1974) et en particulier chez les poissons (Viola et al., 1983; Wilson et Poe, 1985) ont montré que les inhibiteurs trypsiques présents dans les farines de soja était partiellement responsables d'une baisse de l'activité enzymatique digestive, d'une hypertrophie pancréatique et d'une diminution de la croissance. Dans le cas des crustacés pénéides, l'incorporation d'inhibiteurs purifiés et non de farine brute provoque des symptômes identiques (Van Wormhoudt, 1986). Dans le cadre de notre étude, une incorporation trop importante de farine de soja ne provoque pas de variations du taux de conversion, du RHS, de la teneur en protéine de l'hépatopancréas et de la valeur des activités enzymatiques digestives. Des résultats récents (Zwilling et al., 1981; Galgani, 1987) ont montré que l'équipement protéolytique des crustacés était caractérisé par la présence de trypsine, de collagénase et d'une protéase de petit poids moléculaire dont le rôle est de compléter l'ensemble des endoprotéases. Cette protéase possède une spécificité très large et est capable de dégrader l'inhibiteur trypsique du soja (Zwilling et al., 1981). Par ailleurs, au cours de la fabrication, divers éléments dont certains inhibiteurs de la trypsine peuvent être détruits. Dans ces conditions, l'absence d'hypertrophie de l'hépatopancréas et les valeurs constantes d'activité enzymatiques démontrent que l'effet d'une forte quantité de soja se traduit par une baisse de la quantité de nourriture ingérée plutôt que par une action au niveau de l'assimilation de la nourriture avec un effet sur l'activité des enzymes digestives. Par ailleurs, l'utilisation d'un concentré de soja en remplacement du tourteau ne modifie pas les résultats zootechniques. Divers auteurs (Van Wormhoudt, 1980) ont proposé d'utiliser l'indice amylase/protéase pour caractériser la présence de matières végétales dans le régime alimentaire. Les résultats obtenus dans notre étude ne montrent pas de relations entre le taux de tourteau de soja dans l'aliment et la valeur de l'indice amylase/protéase. Les résultats obtenus avec la farine de poisson sont similaires à ceux obtenus avec le soja. Une forte incorporation de farine provoque une baisse de croissance. Pour les mêmes raisons que dans l'expérience concernant le soja, les résultats ne semblent pas liés à la présence de facteurs antinutritionnels mais à un problème d'ingestion. Ce résultat est valable quelle que soit l'origine de la farine de poisson utilisée. Différents facteurs peuvent affecter l'absorption de protéines: l'appétence, la digestibilité ainsi que la composition en acides aminés. Le niveau d'analyse utilisé pour nos expériences ne permet pas toutefois de caractériser l'effet d'un ou des trois de ces facteurs. L'ensemble des résultats permet de conclure que l'incorporation de soja et de farine de poisson dans les aliments doit se faire à faible taux et ce seulement de manière à diversifier les sources protéiques de l'aliment. Les seuils maximaux d'incorporation de ces ingrédients ne doivent pas dépasser $10 \%$. Le problème reste cependant à reconsidérer dans le cas de l'utilisation d'autres sources protéiques et dans le cas du traitement des ingrédients de base qui en augmenterait la valeur alimentaire. L'utilisation récente de concentré de protéines solubles de poisson dans l'alimentation des crustacés d'élevage confirme cette hypothèse. Dans ce cas, une augmentation de la digestibilité due à la solubilisation et à la prédigestion des protéines est observée.

\section{Remerciement}

Les recherches dont il est rendu compte dans cette publication ont été supportées par une aide à la recherche de la CORDET, que nous remercions ici. Une grande partie du support logistique a été assurée par le Centre Océanologique du Pacifique; nous en remercions ici très cordialement la direction et le personnel scientifique et technique.

\section{RÉFÉRENCES}

AQUACOP, 1977. Observations sur la reproduction et la maturation en captivité des crevettes Pénéides en milieu tropical. Actes Colloq. CNEXO, 4, 157-158.

AQUACOP, 1979. Équipements pour fabriquer des granulés par voie humide destinés aux animaux marins. Proc. World Symp. on finish nutrition and fish feed technology, vol. 2, Hamburg, J. Halues, K. Tiews, éd., 143-155.

Bernfeld P., 1955. Sur une méthode de dosage des amylases. In: Methods in Enzymology, Colowick, Kaplan éd., Academic Press, New York, 1, 149-154.
Boghen A. D., J. D. Castell, 1981. Nutritional value of different dietary proteins to juvenile lobsters, Homarus americanus. Aquaculture, 22, 343-351.

Ceccaldi H. J., 1986. La nutrition des crustacés. Oceanis, 12, 31-49.

Charney J., R. Tomarelli, 1947. A coloric method for the determination of the proteolytic activity of duodenal juice. J. Biol. Chem., 171, 501-505.

Dall W., D. Moriarty, 1983. Biology of crustacea, vol. 5. Academic Press, 215-261.

Fenucci J. L., A. Fenucci, A. Lawrence, L. P. Zein-Eldin, 1982. The assimilation of protein and carbohydrate from 
prepared diets by the shrimp, Penaeus stylirostris. $J$. World maricul. Soc., 13, 134-145.

Galgani F., 1987. Digestion des protéines chez les crustacés décapodes. Aspects biochimiques et physiologiques. Thèse dr. État, Aix-Marseille-II, $90 \mathrm{p}$.

Galgani F., H. J. Ceccaldi, AQUACOP. Influence de la nature des protéines de l'aliment sur la croissance et sur les enzymes disgestives de Penaeus monodon. Aquaculture (sous presse).

Gibson R., D. Barker, 1979. The decapod hepatopancreas. Oceanol. Mar. Biol. Ann. Rev., 17, 285-346.

Hoyle R. J., 1973. Digestive Enzyme Secretion After dietary variations in the american lobster (Homaras americanus). J. Fish. Res. Board Can., 30, 1647-1653.

Hummel B., 1959. A modified spectrophotometric determination of chymo-trypsin, trypsin and thrombin. Can. $J$. Biochem. Physiol., 34, 137-142.

Kanazawa A., M. Shimaya, M. Kawasaki, K. Kashiwada, 1970. Nutritional requirements of prawn. II: Requirements for sterols. Bull. Jap. Soc. Sci. Fish., 37, 211-215.

Lee P., L. Smith, A Lawrence, 1984. Digestive protease of Penaeus vannamei Boone: relationship between enzyme activity, size and diet. Aquaculture, 42, 225-239.
Lowry O., N. Rosebrough, A. Fais, R. Randall, 1981. Protein measurement with the Folin phenol reagent. $J$. Biol. Chem., 193. 265-275.

Maugle P. D., O. Deshimara, T. Katayama, K. Simpson, 1982. Effect of short-necked clam Diets on shrimp Growth and digestive enzyme activities. Bull. Jap. Soc. Sci. Fish., 48, 1759-1764.

Rackis J. J., 1974. Biological and Physicological factors in Soybean. J. Am. Oil. Chem. Soc., 51, 162-174.

Shigueno S., 1975. Shrimp culture in Japan. Association for international technical promotion, Tokyo, Japan.

Viola J., S. Mokady, Y. Aricle, 1983. Effects of soybean processing method on the growth of carps (Cyprinuscarpio). Aquaculture, 32, 27-38.

Van Wormhoudt A., 1980. Thèse dr. État, Univ. AixMarseille-II, 351 p. Adaptation des activités digestives de leur cycle et de leur contrôle aux facteurs du milieu chez Palaemon serratus.

Van Wormhoudt A., E. Cruz, J. Guillaume, P. Favrel, 1986. Action de l'inhibiteur typique de soja sur la croissance et l'activité des enzymes digestives chez Penaeus japonicus: rôle éventuel des hormones gastrointestinales. Oceanis, 12, 305-319.

Venkataramiah A., G. J. Lakshmi, G. Gunter, 1975. Effect of protein level and vegetable matter on growth and food conversion efficient of brown shrimp. Aquaculture, 6, 115-125. 